\title{
Integral and differential equations for conformal mapping of bounded multiply connected regions onto a disk with circular slits
}

\author{
Ali H.M. Murid ${ }^{1}$, Ali W. Kareem Sangawi ${ }^{1}$ and M.M.S. Nasser ${ }^{2}$, \\ ${ }^{1}$ Ibnu Sina Institute for Fundamental Science Studies, Universiti Teknologi Malaysia, 81310 UTM Skudai Johor, Malaysia. \\ ${ }^{2}$ Department of Mathematics, Faculty of Science, Ibb University, P. O. Box 70270, Ibb, Yemen. \\ Received 25 December 2010, Revised 5 January 2011, Accepted 10 January 2011, Available online 21 Januari 2011.
}

\begin{abstract}
Conformal mapping is a useful tool in science and engineering. On the other hand exact mapping functions are unknown except for some special regions. In this paper we present a new boundary integral equation with classical Neumann kernel associated to $f^{\prime} / f$, where $f$ is a conformal mapping of bounded multiply connected regions onto a disk with circular slit domain. This boundary integral equation is constructed from a boundary relationship satisfied by a function analytic on a multiply connected region. With $f^{\prime} / f$ known, one can then treat it as a differential equation for computing $f$.
\end{abstract}

| Conformal mapping | Boundary integral equations | Multiply connected regions | Boundary relationship | Differential equations |

\section{INTRODUCTION}

Integral equation methods for conformal mapping of multiply connected regions is currently still a subject of importance. Nehari [1, p. 334] described the five types of slit region as important canonical domain regions for multiply connected regions. They are the parallel slit region, the circular slit region, the radial slit region, the disk with concentric circular slits, and the annulus with concentric circular slits. In general the exact mapping functions are unknown except for some special regions.

Several methods for numerical approximation for the conformal mapping of multiply connected regions have been proposed in $[2,3,4,5,6,7,8$, 9]. Recently, reformulations of conformal mappings from the bounded multiply connected region onto a previous five canonical slit domains as a Riemann-Hilbert problem are discussed in Nasser [10]. Murid and $\mathrm{Hu}$ [11] formulated an integral equation method based on the multiply connected Neumann kernel for conformal mapping of bounded multiply connected regions onto a disk with circular slit but the boundary integral equation involved the unknown circular radii.

In this paper we describe an integral equation method for computing the conformal mapping of multiply connected regions onto a disk with circular slits.

Corresponding author at: Ibnu Sina Institute for Fundamental Science Studies, Universiti Teknologi Malaysia, 81310 UTM Skudai Johor, Malaysia. E-mail addresses: alihassan@utm.my (Ali H.M. Murid)
This boundary integral equation is constructed from a boundary relationship satisfied by a function analytic on a multiply connected region.

The plan of the paper is as follows: After presentation of some auxiliary material in Section 2, we derive in Section 3 a boundary integral equation satisfied by $f^{\prime} / f$, where $f$ is a conformal mapping of bounded multiply connected regions onto a disk with circular slit domain. Section 4 give a numerical implementation for computing $f^{\prime} / f$. In Section 5, we give three examples for verifying our boundary integral equation that was given in Section three. Finally, Section 6 presents a short conclusion.

\section{NOTATIONS AND AUXILIARY MATERIAL}

Let $\Omega$ be a bounded multiply connected region of connectivity $M+1$. The boundary $\Gamma$ consists of $M+1$ smooth Jordan curves $\Gamma_{0}, \Gamma_{1}, \ldots, \Gamma_{M}$ such that $\Gamma_{1}, \ldots, \Gamma_{M}$ lies in the interior of $\Gamma_{0}$, where the outer curve $\Gamma_{0}$ has counterclockwise orientation while the inner curves $\Gamma_{1}, \ldots, \Gamma_{M}$ have clockwise orientation. The positive direction of the contour $\Gamma=\Gamma_{0} \cup \Gamma_{1} \cup \cdots \cup \Gamma_{M}$ is usually that for which $\Omega$ is on the left as one traces the boundary (see Figure 1).

The unit tangent to $\Gamma$ at $z$ is denoted by $T(z)=z^{\prime} /\left|z^{\prime}\right|$, and if $f$ is analytic function which maps $\Omega$ conformally onto a disk with circular slit, then we have the boundary relationship [11, p. 1126]. 
$f(z)^{2}=-|f(z)|^{2} T(z)^{2} \frac{f^{\prime}(z)^{2}}{\left|f^{\prime}(z)\right|^{2}}, z \in \Gamma$.

Suppose that $k$ is a complex constant, $Q(z)$ and $H(z)$ are complex-valued functions defined on $\Gamma$ such that $H(z) \neq 0, Q(z) \neq 0$ and $\overline{H(z)} /(T(z) Q(z))$ satisfies the Holder condition on $\Gamma$. Then the interior relationship is defined as follows:

A complex-valued function $P(z)$ is said to satisfy the interior relationship if $P(z)$ is analytic in $\Omega$ and satisfies the non-homogeneous boundary relationship

$$
P^{+}(z)=k \frac{\overline{T(z) Q(z)}}{\overline{G(z)}} \overline{P^{+}(z)}+\overline{H(z)}, z \in \Gamma
$$

where $G(z)$ is a complex-valued function, analytic in $\Omega$, Holder continuous on $\Gamma$, and $G(z) \neq 0$ on $\Gamma$.
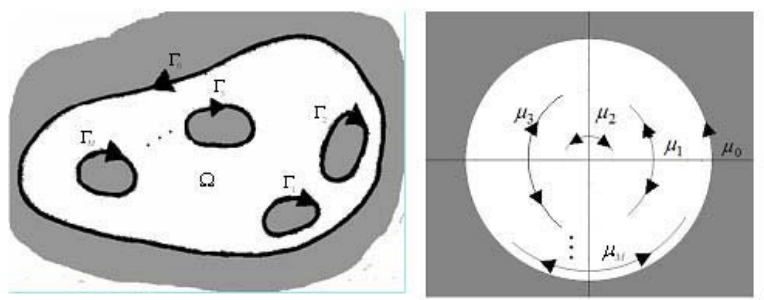

Figure 1. Mapping of the bounded multiply connected region $\Omega$ of connectivity $M+1$ onto a disk with circular slits.

The following theorem gives an integral equation for an analytic function satisfying the interior relationship (2) [12, p. 45].

\section{Theorem 1:}

Let $z \in \Gamma, U(z)$ and $V(z)$ be any complex-valued functions that are defined on $\Gamma$. If the function $P(z)$ satisfies the interior relationship, then

$$
\begin{aligned}
& \frac{1}{2}\left[V(z)+\frac{U(z)}{T(z) Q(z)}\right] P^{+}(z)+P V \int_{\Gamma} K(z, w) P^{+}(w)|d w| \\
& +k U(z)\left[\sum_{j=1}^{M} \operatorname{Re}_{w=a_{j}} \frac{\overline{P(w)}}{(\bar{w}-\bar{z}) \overline{G(w)}}\right]=-U(z) \overline{L_{R}^{-}(z)},
\end{aligned}
$$

where

$$
K(z, w)=\frac{1}{2 \pi i}\left[\frac{U(z)}{(\bar{w}-\bar{z}) \overline{Q(w)}}-\frac{V(z) T(w)}{(w-z)}\right]
$$

$$
\begin{aligned}
L_{R}^{-}(z)=\frac{-1}{2} & \frac{H(z)}{Q(z) T(z)} \\
& +P V \frac{1}{2 \pi i} \int_{\Gamma} \frac{H(w)}{(w-z) Q(w) T(w)} d w,
\end{aligned}
$$

and the sum is over all those zeros $a_{1}, a_{2}, \ldots, a_{M}$ of $G$ that lie inside $\Omega$. If $G$ has no zeros in $\Omega$, then the term containing the residue in (3) will not appear.

\section{THE BOUNDARY INTEGRAL EQUATION FOR CONFORMAL MAPPING OF MULTIPLY CONNECTED REGIONS}

This section gives an application of Theorem 1 to conformal mapping of multiply connected regions to disk with circular slit domain. Let $f(z)$ be the mapping function which maps the domain $\Omega$ in the $z$-plane onto a canonical domain of the disk with circular slit domain in the $w$-plane. The function $f$ is made uniquely determined by prescribing that $f(a)=0$ and $f^{\prime}(a)>0$. Thus the function $f$ can be written as [10, p. 134]

$f(z)=(z-a) e^{(z-a) h(z)-\alpha}$,

where $h$ is an analytic in $\Omega$ and $\alpha$ is real constant.

Taking the derivative of both sides of the equation (6) and dividing both sides by $f$ yields

$\frac{f^{\prime}(z)}{f(z)}=\frac{1}{z-a}+h(z)+(z-a) h^{\prime}(z)$. Then

$D(z)=\frac{f^{\prime}(z)}{f(z)}-\frac{1}{z-a}=h(z)+(z-a) h^{\prime}(z)$ is analytic in $\Omega$.

Thus

$\frac{f^{\prime}(z)}{f(z)}=D(z)+\frac{1}{z-a}$.

Note that equation (1) can be written in the following form

$\left|\frac{f^{\prime}(z)}{f(z)}\right|^{2}=-T(z)^{2}\left(\frac{f^{\prime}(z)}{f(z)}\right)^{2}, z \in \Gamma$.

From equations (7) and (8), and after some arrangement yields

$D(z)=-\bar{T}(z)^{2} \overline{D(z)}-\frac{\bar{T}(z)^{2}}{\bar{z}-\bar{a}}-\frac{1}{z-a}, \quad z \in \Gamma$ 
Comparison of equation (2) and equation (9) leads to a choice of $P^{+}(z)=D(z), \quad k=-1, Q(z)=T(z), \quad G(z)=1$, $H(z)=-\frac{T(z)^{2}}{z-a}-\frac{1}{\bar{z}-\bar{a}}, \quad U(z)=\bar{T}(z) \bar{Q}(z)$ and $\quad V(z)=1$. Substituting these assignments into (3) leads to an integral equation,

$$
\begin{gathered}
D(z)+P V \frac{1}{2 \pi i} \int_{\Gamma}\left[\frac{\bar{T}(z)^{2}}{(\bar{w}-\bar{z}) \bar{T}(w)}-\frac{T(w)}{w-z}\right] D(w)|d w| \\
=-\bar{T}(z)^{2} \overline{L^{-}{ }_{R}}(z), \quad z \in \Gamma .
\end{gathered}
$$

Note that $G(z)$ does not have any zeros in $\Omega$ because $G(z)$ is a constant function so the term containing the residue does not appear in (10). Multiply both sides of (10) by $T(z)$ and using the fact that $T(z) \bar{T}(z)=|T(z)|^{2}=1$, gives

$$
\begin{gathered}
T(z) D(z)+P V \frac{1}{2 \pi i} \int_{\Gamma}\left[\frac{\bar{T}(z)}{(\bar{w}-\bar{z})}-\frac{T(z)}{w-z}\right] D(w) T(w)|d w| \\
=-\bar{T}(z) \overline{L^{-}}(z), \quad z \in \Gamma,
\end{gathered}
$$

Where

$$
\begin{aligned}
T(z) L_{R}^{-}(z)= & \frac{1}{2}\left[\frac{T(z)}{z-a}+\frac{\bar{T}(z)}{\bar{z}-\bar{a}}\right]- \\
& T(z) P V \frac{1}{2 \pi i} \int_{\Gamma} \frac{1}{(w-z)(w-a)} d w- \\
& T(z) P V \frac{1}{2 \pi i} \int_{\Gamma} \frac{\bar{T}(w)^{2}}{(w-z)(\bar{w}-\bar{a})} d w, z \in \Gamma .
\end{aligned}
$$

Since

$$
\frac{1}{(w-a)(w-z)}=\frac{1}{z-a}\left[\frac{1}{w-z}-\frac{1}{w-a}\right]
$$

and for $z \in \Gamma, a \in \Omega$ by [4, p. 91]

$$
\begin{aligned}
& \frac{1}{2 \pi i} \int_{\Gamma} \frac{1}{w-z} d w=\frac{1}{2}, \frac{1}{2 \pi i} \int_{\Gamma} \frac{1}{w-a} d w=1 . \text { Thus } \\
& P V \frac{1}{2 \pi i} \int_{\Gamma} \frac{1}{(w-z)(w-a)} d w=-\frac{1}{2(z-a)} .
\end{aligned}
$$

Substituting this result into equation (12) and using the fact that $\bar{T}(w) d w=|d w|$, gives
$T(z) L_{R}^{-}(z)=\frac{T(z)}{z-a}+\frac{1}{2} \frac{\bar{T}(z)}{\bar{z}-\bar{a}}-$

$$
T(z) P V \frac{1}{2 \pi i} \int_{\Gamma} \frac{\bar{T}(w)}{(w-z)(\bar{w}-\bar{a})}|d w|, z \in \Gamma .
$$

Let $F(z)=\frac{f^{\prime}(z)}{f(z)}$. Then $D(z)=F(z)-\frac{1}{z-a}$.

From equations (11), (13), and (14) we get

$$
\begin{gathered}
T(z) F(z)-\frac{1}{2} \frac{T(z)}{z-a}+P V \frac{1}{2 \pi i} \int_{\Gamma}\left[\frac{\bar{T}(z)}{(\bar{w}-\bar{z})}-\frac{T(z)}{w-z}\right] T(w) \\
\times F(w)|d w|+T(z) P V \frac{1}{2 \pi i} \int_{\Gamma} \frac{T(w)}{(w-z)(w-a)}|d w| \\
=-\frac{\bar{T}(z)}{\bar{z}-\bar{a}}, z \in \Gamma .
\end{gathered}
$$

Also by using [4, p. 91] and the fact that $T(w)|d w|=d w$, gives

$P V \frac{1}{2 \pi i} \int \frac{T(w)}{\Gamma}|d w|=-\frac{1}{2} \frac{1}{(z-a)(w-a)}$.

Substituting this result into equation (15) yields

$$
\begin{array}{r}
T(z) F(z)+P V \frac{1}{2 \pi i}\left[\int \frac{\bar{T}(z)}{(\overline{\bar{w}}-\bar{z})}-\frac{T(z)}{w-z}\right] T(w) F(w)|d w| \\
=\frac{T(z)}{z-a}-\frac{\bar{T}(z)}{\bar{z}-\bar{a}}, \quad z \in \Gamma .
\end{array}
$$

The above integral equation can also be written briefly as

$$
F_{1}(z)+\int_{\Gamma} N(z, w) F_{1}(w)|d w|=\frac{T(z)}{z-a}-\frac{\bar{T}(z)}{\bar{z}-\bar{a}}, z \in \Gamma,
$$

where

$$
\begin{gathered}
F_{1}(z)=T(z) F(z), \\
N(z, w)=\left\{\begin{array}{cc}
\frac{1}{\pi} \operatorname{Im}\left[\frac{T(z)}{z-w}\right], & \text { if } z, w \in \Gamma, z \neq w, \\
\frac{1}{2 \pi} \frac{\operatorname{Im}\left[z^{\prime \prime}(t) \overline{z^{\prime}(t)}\right]}{\left|z^{\prime}(t)\right|^{3}}, & \text { if } \quad z=w \in \Gamma .
\end{array}\right.
\end{gathered}
$$

The kernel $N$ is also known as the Neumann kernel. Note that the PV symbol is no longer required in (16) since the integrand is continuous along the path of integration. Since $\lambda=-1$ is an eigenvalue of $N$ with multiplicity $M$, by Theorem 12 in $[13$, p.53] the integral equation (16) is not 
uniquely solvable. So we need to modify the integral equation to solve it numerically.

Note that $a \in \Omega$ and $\frac{f^{\prime}(z)}{f(z)}=\frac{1}{z-a}+h(z)+(z-a) h^{\prime}(z)$ so by using the fundamental theorem $[14$, p. 164] and the fact that $T(w)|d w|=d w$, gives

$$
\int_{-\Gamma_{1}} \frac{1}{2 \pi} F_{1}(w)|d w|=0,
$$

By using the same manner we can show that

$$
\int_{-\Gamma_{2}} \frac{1}{2 \pi} F_{1}(w)|d w|=0, \ldots, \int_{-\Gamma_{M}} \frac{1}{2 \pi} F_{1}(w)|d w|=0 .
$$

We can combine the conditions (17) and (18) in the following form

$$
\int_{-\Gamma_{q}} \frac{1}{2 \pi} F_{1}(w)|d w|=0, \quad q=1,2, \ldots, M .
$$

Thus the integral equation (16) with the condition (19) has a unique solution.

By solving the integral equations (16) and (19) simultaneously for $F_{1}$, we can obtain the function $F$ and hence determine $D$. With $D$ known, one can then treat (7) as a first order ordinary differential equation for computing $f$, as follows

$$
f^{\prime}(z)=\left[D(z)+\frac{1}{z-a}\right] f(z) .
$$

After finding $f$ we can calculate the radii by taking the modulus for $f$.

\section{NUMERICAL IMPLEMENTATION}

In this section we first describe in detail a numerical method for computing the mapping function $F_{1}$ for the case of doubly connected region. Using the parameterizations $z_{0}(t)$ of $\Gamma_{0}$ for $t: 0 \leq t \leq \beta_{0}$ and $z_{1}(t)$ of $-\Gamma_{1}$ for $t: 0 \leq t \leq \beta_{1}$ the system of integral equations (16) and (19) become

$$
\begin{gathered}
F_{1}\left(z_{0}(t)\right)+\int_{0}^{\beta_{0}} N\left(z_{0}(t), z_{0}(s)\right) F_{1}\left(z_{0}(s)\right)\left|z_{0}^{\prime}(s)\right| d s \\
-\int_{0}^{\beta_{1}}\left(N\left(z_{0}(t), z_{1}(s)\right)-\frac{1}{2 \pi}\right) F_{1}\left(z_{1}(s)\right)\left|z_{1}^{\prime}(s)\right| d s \\
=2 i \operatorname{Im}\left[\frac{T\left(z_{0}(t)\right)}{z_{0}(t)-a}\right], z_{0}(t) \in \Gamma_{0},
\end{gathered}
$$

$$
\begin{gathered}
F_{1}\left(z_{1}(t)\right)+\int_{0}^{\beta_{0}} N\left(z_{1}(t), z_{0}(s)\right) F_{1}\left(z_{0}(s)\right)\left|z_{0}^{\prime}(s)\right| d s \\
-\int_{0}^{\beta_{1}}\left(N\left(z_{1}(t), z_{1}(s)\right)-\frac{1}{2 \pi}\right) F_{1}\left(z_{1}(s)\right)\left|z_{1}^{\prime}(s)\right| d s \\
=2 i \operatorname{Im}\left[\frac{T\left(z_{1}(t)\right)}{z_{1}(t)-a}\right], z_{1}(t) \in \Gamma_{1} .
\end{gathered}
$$

Multiply both sides of equations (21) and (22) by $\left|z_{0}^{\prime}(t)\right|$ and $\left|z_{1}^{\prime}(t)\right|$ respectively, yields

$$
\begin{aligned}
& \left|z_{0}^{\prime}(t)\right| F_{1}\left(z_{0}(t)\right)+\int_{0}^{\beta_{0}}\left|z_{0}^{\prime}(t)\right| N\left(z_{0}(t), z_{0}(s)\right) F_{1}\left(z_{0}(s)\right) \\
& \times\left|z_{0}^{\prime}(s)\right| d s-\int_{0}^{\beta_{1}}\left|z_{0}^{\prime}(t)\right|\left(N\left(z_{0}(t), z_{1}(s)\right)-\frac{1}{2 \pi}\right) F_{1}\left(z_{1}(s)\right) \\
& \times\left|z_{1}^{\prime}(s)\right| d s=2 i\left|z_{0}^{\prime}(t)\right| \operatorname{Im}\left[\frac{T\left(z_{0}(t)\right)}{z_{0}(t)-a}\right], z_{0}(t) \in \Gamma_{0}, \\
& \left|z_{1}^{\prime}(t)\right| F_{1}\left(z_{1}(t)\right)+\int_{0}^{\beta_{0}}\left|z_{1}^{\prime}(t)\right| N\left(z_{1}(t), z_{0}(s)\right) F_{1}\left(z_{0}(s)\right) \\
& \times\left|z_{0}^{\prime}(s)\right| d s-\int_{0}^{\beta_{1}}\left|z_{1}^{\prime}(t)\right|\left(N\left(z_{1}(t), z_{1}(s)\right)-\frac{1}{2 \pi}\right) F_{1}\left(z_{1}(s)\right) \\
& \times\left|z_{1}^{\prime}(s)\right| d s=2 i\left|z_{1}^{\prime}(t)\right| \operatorname{Im}\left[\frac{T\left(z_{1}(t)\right)}{z_{1}(t)-a}\right], z_{1}(t) \in \Gamma_{1}, \quad(24)
\end{aligned}
$$

Defining

$$
\begin{aligned}
& \phi_{0}(t)=\left|z_{0}^{\prime}(t)\right| F_{1}\left(z_{0}(t)\right), \quad \phi_{1}(t)=\left|z_{1}^{\prime}(t)\right| F_{1}\left(z_{1}(t)\right), \\
& K_{00}\left(t_{0}, s_{0}\right)=\left|z_{0}^{\prime}(t)\right| N\left(z_{0}(t), z_{0}(s)\right), \\
& K_{01}\left(t_{0}, s_{1}\right)=\left|z_{0}^{\prime}(t)\right|\left(N\left(z_{0}(t), z_{1}(s)\right)-\frac{1}{2 \pi}\right), \\
& K_{10}\left(t_{1}, s_{0}\right)=\left|z_{1}^{\prime}(t)\right| N\left(z_{1}(t), z_{0}(s)\right), \\
& K_{11}\left(t_{1}, s_{1}\right)=\left|z_{1}^{\prime}(t)\right|\left(N\left(z_{1}(t), z_{1}(s)\right)-\frac{1}{2 \pi}\right),
\end{aligned}
$$


$\psi_{0}(t)=2 i\left|z_{0}^{\prime}(t)\right| \operatorname{Im}\left[\frac{T\left(z_{0}(t)\right)}{z_{0}(t)-a}\right]$

$\psi_{1}(t)=2 i\left|z_{1}^{\prime}(t)\right| \operatorname{Im}\left[\frac{T\left(z_{1}(t)\right)}{z_{1}(t)-a}\right]$,

the system of equations (23) and (24) can be briefly written as

$$
\begin{array}{r}
\phi_{0}(t)+\int_{0}^{\beta_{0}} K_{00}\left(t_{0}, s_{0}\right) \phi_{0}(s) d s-\int_{0}^{\beta_{1}} K_{01}\left(t_{0}, s_{1}\right) \phi_{1}(s) d s \\
=\psi_{0}(t), \\
\phi_{1}(t)+\int_{0}^{\beta_{0}} K_{10}\left(t_{1}, s_{0}\right) \phi_{0}(s) d s-\int_{0}^{\beta_{1}} K_{11}\left(t_{1}, s_{1}\right) \phi_{1}(s) d s \\
=\psi_{1}(t) .
\end{array}
$$

Since the functions $\phi$ and $K$ in the above systems are $\beta$-periodic, a reliable procedure for solving equations (25) and (26) numerically is by using the Nyström's method [15] with the trapezoidal rule. The trapezoidal rule is the most accurate method for integrating periodic functions numerically [16, pp. 134-142]. We choose $\beta_{0}=\beta_{1}=2 \pi$ and $n$ equidistant collocation points $t_{i}=(i-1) \beta_{0} / n, \quad 1 \leq i \leq n \quad$ on $\Gamma_{0} \quad$ and $m$ equidistant collocation points $t_{\widetilde{i}}=(\tilde{i}-1) \beta_{1} / m, \quad 1 \leq \tilde{i} \leq m \quad$ on $\Gamma_{1}$. Applying the Nyström's method with trapezoidal rule to discretize equations (25) and (26), we obtain

$$
\begin{gathered}
\phi_{0}\left(t_{i}\right)+\frac{\beta_{0}}{n} \sum_{j=1}^{n} K_{00}\left(t_{i}, t_{j}\right) \phi_{0}\left(t_{j}\right)-\frac{\beta_{1}}{m} \sum_{\tilde{j}=1}^{m} K_{01}\left(t_{i}, t_{\widetilde{j}}\right) \phi_{1}\left(t_{\widetilde{j}}\right) \\
=\psi_{0}\left(t_{i}\right), \\
\begin{aligned}
\phi_{1}\left(t_{\widetilde{i}}\right)+\frac{\beta_{0}}{n} \sum_{j=1}^{n} K_{10}\left(t_{\widetilde{i}}, t_{j}\right) \phi_{0}\left(t_{j}\right)-\frac{\beta_{1}}{m} \sum_{\tilde{j}=1}^{m} K_{11}\left(t_{\widetilde{i}}, t_{\widetilde{j}}\right) \phi_{1}\left(t_{\widetilde{j}}\right) \\
=\psi_{1}\left(t_{\widetilde{i}}\right),
\end{aligned}
\end{gathered}
$$

Equations (27) and (28) lead to a system of $(n+m)$ nonhomogeneous linear complex equations in $n$ unknowns $\phi_{0}\left(t_{i}\right), m$ unknowns $\phi_{1}\left(t_{\widetilde{i}}\right)$. By defining the matrices

$$
\begin{array}{ll}
B_{i j}=\frac{\beta_{0}}{n} K_{00}\left(t_{i}, t_{j}\right), & C_{i \widetilde{j}}=\frac{\beta_{1}}{m} K_{01}\left(t_{i}, t_{\widetilde{j}}\right), \\
D_{\widetilde{i} j}=\frac{\beta_{0}}{n} K_{10}\left(t_{\widetilde{i}}, t_{j}\right), & E_{\widetilde{i} \widetilde{j}}=\frac{\beta_{1}}{m} K_{11}\left(t_{\widetilde{i}}, t_{\tilde{j}}\right), \\
\mathrm{x}_{0 \mathrm{i}}=\phi_{0}\left(t_{i}\right), & \mathrm{x}_{1 \widetilde{\mathrm{i}}}=\phi_{1}\left(t_{\widetilde{i}}\right),
\end{array}
$$

$$
b_{0 i}=\psi_{0}\left(t_{i}\right), \quad b_{1 \widetilde{i}}=\psi_{1}\left(t_{\widetilde{i}}\right),
$$

the system of equations (27) and (28) can be written as $(n+m)$ by $(n+m)$ system of equations

$\left[I_{n n}+B_{n n}\right] \mathrm{x}_{0 \mathrm{n}}-C_{n m} \mathrm{x}_{1 \mathrm{~m}}=b_{0 n}$,

$D_{m n} \mathrm{x}_{0 \mathrm{n}}+\left[I_{m m}-E_{m m}\right] \mathrm{x}_{1 \mathrm{~m}}=b_{1 m}$.

The result in matrix form for the system of equations (29) and (30) is

$\left(\begin{array}{cc}I_{n n}+B_{n n} & -C_{n m} \\ D_{m n} & I_{m m}-E_{m m}\end{array}\right)\left(\begin{array}{l}\mathrm{x}_{0 \mathrm{n}} \\ \mathrm{x}_{1 \mathrm{~m}}\end{array}\right)=\left(\begin{array}{l}b_{0 n} \\ b_{1 m}\end{array}\right)$,

Defining

$\mathrm{A}=\left(\begin{array}{cc}I_{n n}+B_{n n} & -C_{n m} \\ D_{m n} & I_{m m}-E_{m m}\end{array}\right), \mathrm{x}=\left(\begin{array}{c}\mathrm{x}_{0 \mathrm{n}} \\ \mathrm{x}_{1 \mathrm{~m}}\end{array}\right)$ and

$b=\left(\begin{array}{l}b_{0 n} \\ b_{1 m}\end{array}\right)$,

the $(n+m)$ by $(n+m)$ system can be written briefly as $\mathrm{Ax}=b$.

\section{NUMERICAL EXPERIMENT}

For numerical experiments, we have used three test regions based on the examples given in $[5,17,18]$. All the computations are done using MATHEMATICA 7.1 (16 digit machine precision).

The test regions are annulus, circular frame and frame of Limacon. $N$ number of collocation points on each boundary has been chosen. The sub-norm error results between exact values for $f^{\prime} / f$ and their approximations $\left(f^{\prime} / f\right)_{n}$ are shown in Tables 1,2 and 3.

\section{Example 1 Annulus:}

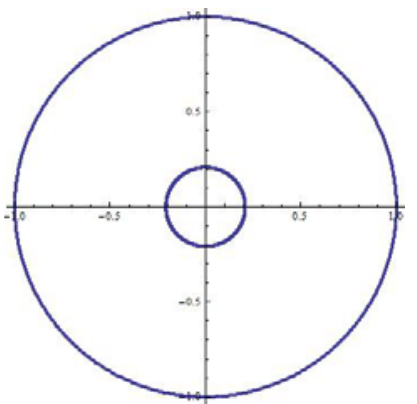

Figure 1. Annulus: with $\tau=0.5$ and $\widetilde{r}=q=e^{-\pi \tau}$. 
Consider a frame of circular annulus $A=\{z: \widetilde{r}<|z|<1\}$, $\widetilde{r}=q=e^{-\pi \tau}, \tau>0$.

$\Gamma_{0}:\{z(t)=\cos t+i \sin t\}$,

$\Gamma_{1}:\{z(t)=\tilde{r}(\cos t+\sin t)\}$.

The exact mapping function is given by [17]

$$
f(z)=-e^{2 \sigma} \frac{\theta_{4}\left(\frac{1}{2 i} \log z+\frac{i \pi \tau}{2}-i \sigma\right)}{\theta_{4}\left(\frac{1}{2 i} \log z+\frac{i \pi \tau}{2}+i \sigma\right)}, 0<\sigma<\frac{\pi \tau}{2},
$$

with $\mu=e^{-2 \sigma}$ and $\theta_{4}$ being the Jacobi Theta-functions. We have chosen $\tau=0.5, \tilde{r}=e^{-\pi \tau}$ and $\sigma=0.2$. Since $\theta_{4}(\pi \tau i / 2)=0$ [17], this implies $a=e^{-2 \sigma}=\mu$.

Table 1. Error norm (Annulus)

\begin{tabular}{|c|c|}
\hline$n=m$ & $\left\|\frac{f^{\prime}}{f}-\left(\frac{f^{\prime}}{f}\right)_{n}\right\|_{\infty}$ \\
\hline 16 & $1.0(-02)$ \\
\hline 32 & $1.6(-05)$ \\
\hline 64 & $4.6(-11)$ \\
\hline 128 & $1.0(-14)$ \\
\hline
\end{tabular}

Example 2 Circular Frame:

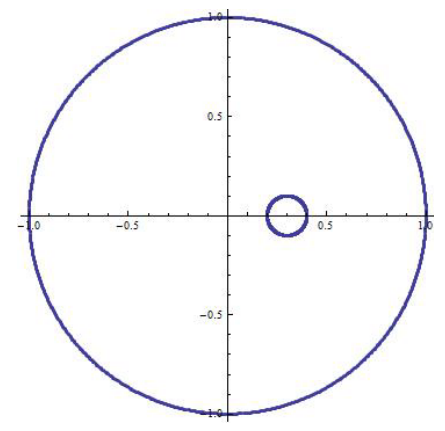

Figure 2. Circular Frame: with $c=0.3, \rho=0.1$.

Consider a pair of Limacon [18]

$\Gamma_{0}:\left\{z(t)=e^{i t}\right\}$

$$
\Gamma_{1}:\left\{z(t)=c+\rho e^{i t}\right\}, t: 0 \leq t \leq 2 \pi,
$$

such that the domain bounded by $\Gamma_{0}$ and $\Gamma_{1}$ is the domain between a unit circle and a circle center at $c$ with radius $\rho$.
Since $\theta_{4}(\pi \tau i / 2)=0$ and $\tilde{r}=q=e^{-\pi \tau}$, this implies $\tau=\frac{\ln (\tilde{r})}{-\pi}$ and $a=\frac{\lambda-e^{-2 \sigma}}{1-\lambda e^{-2 \sigma}}$. We choose a real number $\sigma$ satisfying $0<\sigma<\pi \tau / 2$. Then the exact mapping function is given by

$$
f(z)=-e^{2 \sigma} \frac{\theta_{4}\left(\frac{1}{2 i} \log p(z)+\frac{i \pi \tau}{2}-i \sigma\right)}{\theta_{4}\left(\frac{1}{2 i} \log p(z)+\frac{i \pi \tau}{2}+i \sigma\right)}, 0<\sigma<\frac{\pi \tau}{2},
$$

where $p(z)=\frac{z-\lambda}{\lambda z-1}$ with

$$
\lambda=\frac{2 c}{1+\left(c^{2}-\rho^{2}\right)+\left(\left(1-(c-\rho)^{2}\right)\left(1-(c+\rho)^{2}\right)\right)^{1 / 2}},
$$

$$
\tilde{r}=\frac{2 \rho}{1+\left(c^{2}-\rho^{2}\right)+\left(\left(1-(c-\rho)^{2}\right)\left(1-(c+\rho)^{2}\right)\right)^{1 / 2}} .
$$

Table 2. Error norm (Circular frame) with $c=0.3, \rho=0.1, \sigma=0.5$

\begin{tabular}{|c|c|}
\hline$n=m$ & $\left\|\frac{f^{\prime}}{f}-\left(\frac{f^{\prime}}{f}\right)_{n}\right\|_{\infty}$ \\
\hline 8 & $5.7(-03)$ \\
\hline 16 & $3.3(-06)$ \\
\hline 32 & $1.5(-12)$ \\
\hline 64 & $1.7(-14)$ \\
\hline
\end{tabular}

Example 3 Frame of Limacon:

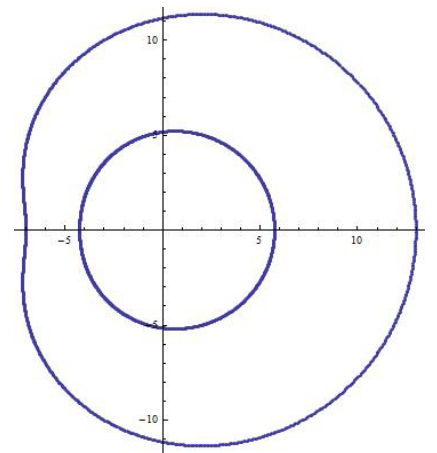

Figure 3. Frame of Limacon: with $a_{0}=10, a_{1}=5, b_{0}=3$ and $b_{1}=b_{0} / 4$.

Consider a pair of Limacon [5, p. 307] 
$\Gamma_{0}:\left\{z(t)=a_{0} \cos t+b_{0} \cos 2 t+i\left(a_{0} \sin t+b_{0} \sin 2 t\right)\right.$,

$$
\begin{array}{r}
\left.a_{0}>0, b_{0}>0\right\}, \\
\Gamma_{1}:\left\{z(t)=a_{1} \cos t+b_{1} \cos 2 t+i\left(a_{1} \sin t+b_{1} \sin 2 t\right),\right.
\end{array}
$$

$$
\left.a_{1}>0, b_{1}>0\right\}
$$

with $a_{0}=10, a_{1}=5, b_{0}=3$ and $b_{1}=b_{0} / 4$ where $t: 0 \leq t \leq 2 \pi$. The value of $a_{0}, a_{1}, b_{0}$ and $b_{1}$ are chosen so that $b_{1} / b_{0}=a_{1} / a_{0}$ and $\tilde{r}=a_{1} / a_{0}$. Since $\theta_{4}(\pi \tau i / 2)=0$ and $\widetilde{r}=q=e^{-\pi \tau}, \quad$ this implies $\quad \tau=\frac{\ln \left(a_{1} / a_{0}\right)}{-\pi} \quad$ and $a=\frac{\left(2 b_{0} e^{-2 \sigma}+a_{0}\right)^{2}-a_{0}^{2}}{4 b_{0}}$. We choose a real number $\sigma$ satisfying $0<\sigma<\pi \tau / 2$. The exact mapping function is given by

$f(z)=-e^{2 \sigma} \frac{\theta_{4}\left(\frac{1}{2 i} \log p(z)+\frac{i \pi \tau}{2}-i \sigma\right)}{\theta_{4}\left(\frac{1}{2 i} \log p(z)+\frac{i \pi \tau}{2}+i \sigma\right)}, 0<\sigma<\frac{\pi \tau}{2}$

where $p(z)=\frac{\left(a_{0}^{2}+4 b_{0} z\right)^{1 / 2}-a_{0}}{2 b_{0}}, \mu=e^{-2 \sigma}$.
Table 3. Error norm (frame of Limacon) with $\sigma=0.1$

\begin{tabular}{|c|c|}
\hline$n=m$ & $\left\|\frac{f^{\prime}}{f}-\left(\frac{f^{\prime}}{f}\right)_{n}\right\|_{\infty}$ \\
\hline 32 & $3.1(-03)$ \\
\hline 64 & $2.0(-06)$ \\
\hline 128 & $5.6(-12)$ \\
\hline 256 & $2.5(-15)$ \\
\hline
\end{tabular}

\section{CONCLUSION}

From this study, we have constructed a new boundary integral equation for conformal mapping of regions of connectivity $M+1$ onto a disk with circular slits. The boundary integral equation for $f^{\prime} / f$ involved the classical Neumann kernel, where $f$ is a conformal mapping of bounded multiply connected regions onto a disk with circular slit domain. The advantage of our method over [11] is that our boundary integral equation does not involve the unknown circular radii. Discretized integral equation leads to a system of linear equations. With $f^{\prime} / f$ known, one can then treat it as a differential equation for computing $f$.

\section{ACKNOWLEDGEMENT}

This work was supported in part by the Malaysian Ministry of Higher Education (MOHE) through the Research Management Centre (RMC), Universiti Teknologi Malaysia (FRGS Vote 78479). This support is gratefully acknowledged.

\section{REFERENCES}

[1] Z. Nehari, Conformal Mapping, Dover Publications, Inc, New York, 1952.

[2] D. Crowdy, and J. Marshall, Computational Methods and Function Theory 6 (2006) 59-76.

[3] S.W. Ellacott, Numerische Mathematik 33 (1979) 437-446.

[4] P. Henrici, Applied and Computational Complex Analysis, Vol. 3, John Wiley, New York, 1974.

[5] P.K. Kythe, Computational Conformal Mapping, Birkhauser Boston, New Orleans, 1998.

[6] A. H. M. Murid and N. A. Mohamed, Int. J. of Pure and Appl. Math. 38(2007), 229-250.

[7] A. H. M. Murid and M. R. M. Razali, Matematika 15 (1999), 79-93.

[8] D. Okano, H. Ogata, K. Amano and M. Sugihara, Journal of Comp. Appl. Math. 159 (2003), 109-117.

[9] G. T. Symm, Numer. Math. 13 (1969), 448-457.

[10] M. M. S. Nasser, Journal of Comput. Methods. Funct. Theory 9 (2009), 127-143.

[11] A. H. M. Murid and Laey-Nee Hu, Int. J. Contemp. Math. Sciences 4 (2009), 1121-1147.

[12] M. M. S. Nasser, Boundary Integral Equation Approach for Riemann Problem, PhD Thesis, Department of Mathematics, Universiti Teknologi Malaysia, 2005.

[13] R. Wegmann and M. M. S. Nasser, J. Comput. Appl. Math. 214 (2008), 36-57.

[14] E. B. Saff and A. D. Snider, Fundamentals of Complex Analysis, Pearson Education, Inc. New Jersey, 2003.

[15] K. E. Atkinson, A Survey of Numerical Methods for the Solution of Fredholm Integral Equations, Society for Industrial and Applied Mathematics, Philadelphia, 1976.

[16] P. J. Davis and P. Rabinowitz, Methods of Numerical Integration, 2nd Edition, Academic Press, Orlando, 1984.

[17] E. T. Whittaker and G. N. Watson, A Course of Modern Analysis, University Press, Cambridge, 1927.

[18] W. van Koppenfels and F. Stallmann, Praxis der Konformen Abbildung, Göttingen, Heidelberge, Berlin, 1959. 